\title{
THE METHOD OF THE HYPERCIRCLE FOR A CLASS OF NONLINEAR EQUATIONS
}

\author{
A. M. ARTHURS and V. G. HART
}

(Received 2 May 1978)

(Revised 18 September 1978)

\begin{abstract}
We present a geometrical method for the solution of a certain class of nonlinear boundary value problems. The results generalize those of the standard hypercircle method for linear problems. Two illustrative examples are described.
\end{abstract}

\section{Introduction}

The hypercircle [7] provides a method for the approximate solution of certain boundary value problems of mathematical physics. In this method the analytical problem of solving a differential equation subject to prescribed boundary conditions is transformed into a geometrical problem of finding the point of intersection of two manifolds in a suitably chosen function space. As developed so far, the theory deals with boundary value problems that are linear, and in this paper we consider an extension which enables us to obtain corresponding geometrical results for an interesting class of non-linear boundary value problems. Our theory is illustrated by calculations for two examples, corresponding to non-linear ordinary and partial differential equations respectively.

\section{Class of non-linear problems}

We shall consider boundary value problems described by equations of the form

$$
\begin{array}{rlrl}
L \phi & \equiv T^{*} T \phi=f(\phi) & \text { in } V, \\
\phi=\phi_{B} & \text { on } B,
\end{array}
$$


where $L=T^{*} T$ is a linear self-adjoint second-order differential operator, $f$ is a given scalar-valued function of a scalar variable $\phi$ with certain properties that will emerge later, and $\phi_{B}$ is a prescribed function on the smooth boundary $B$ of the closed region $V$, a compact connected subset of Euclidean space $E^{n}$. We assume the existence of a unique solution of (1), (2).

Let $H_{u}$ and $H_{\phi}$ be real Hilbert spaces of vectors $u$ and scalars $\phi$ respectively defined on $V+B$, and we denote by $[$,$] and \langle$,$\rangle the inner products on H_{u}$ and $H_{\phi}$. Then let the operator $T$ map $H_{\phi}$ into $H_{u}$ and suppose that the domain of $T$ is dense in $H_{\phi}$. The adjoint operator $T^{*}$ is defined on $H_{u}$ and maps $H_{u}$ into $H_{\phi}$. We have

$$
[u, T \phi]=\left\langle T^{*} u, \phi\right\rangle+\text { boundary terms, }
$$

and we assume that these boundary terms in (3) vanish when $\phi_{B}=0$. Standard examples of the scalar products are

\begin{tabular}{cccc}
\hline$[u, v]$ & $\langle\phi, \psi\rangle$ & $T$ & $T^{*}$ \\
\hline $\int_{a}^{b} u v d x$ & $\int_{a}^{b} \phi \psi d x$ & $d / d x$ & $-d / d x$ \\
$\int_{\nabla} u . v d V$ & $\int_{\nabla} \phi \psi d V$ & $\operatorname{grad}$ & $-\operatorname{div}$ \\
\hline
\end{tabular}

which we shall use later. More general examples arise when the inner products involve weighting factors or when the operators are more complicated, but we do not need the details here.

Examples of operators $T, T^{*}$ and their corresponding domains are given in [1]. For the examples given in this paper it is sufficient to suppose that the domains of $T$ and $T^{*}$ are subsets of continuous functions. We note also that if the function $f$ in (1) satisfies condition (12) following, the existence of a unique solution of the problem (1), (2) follows from consideration of the canonical form of the problem (see $[1$, p. 67] and $[6$, p. 146]; see also the observation after equation (16)).

\section{Geometrical formulation}

To derive hypercircle results for the class of problems in (1) and (2), we first employ the basic procedure of splitting equation (1) into a pair of canonical equations by taking

$$
\begin{gathered}
T \phi=u, \quad \phi=\phi_{B} \text { on } B, \\
T^{*} u=f(\phi) \text { in } V .
\end{gathered}
$$

A solution $(u, \phi)$ of these equations satisfies (1) and (2). 
Next we introduce a new function space ( $F$-space like phase space) with points $S_{i}=\left(u_{i}, \phi_{i}\right)$, but without metric for the moment. Let $S=(u, \phi)$ denote the solution of (5) and (6). Then $S$ is the intersection of two manifolds:

(1) a linear subspace $\Omega_{1}$ defined by

$$
T \phi_{1}=u_{1}, \quad \phi_{1}=\phi_{B} \text { on } B ;
$$

(2) a non-linear manifold $\Omega_{2}$ defined by

$$
T^{*} u_{2}=f\left(\phi_{2}\right) \text { in } V .
$$

Now we shall define the scalar or inner product for any two vectors $S_{i}$ and $S_{j}$ in $F$-space by

$$
S_{i} . S_{j}=\left[u_{i}, u_{j}\right]+\left\langle\phi_{i}, \gamma \phi_{j}\right\rangle,
$$

where $S_{i}=\left(u_{i}, \phi_{i}\right)$ and $\gamma$ is some positive constant. Then the metric is positive definite. Evidently $F$-space is the Cartesian product $H_{u} \times H_{\phi}$, and is a Hilbert space if $\gamma \neq 0$. If $\gamma=0$ we can work instead with the $F$-space $H_{u}$ of vectors $u_{i}$.

If $S=(u, \phi)$ is the solution, $S_{1}=\left(u_{1}, \phi_{1}\right)$ a point in $\Omega_{1}$ and $S_{2}=\left(u_{2}, \phi_{2}\right)$ a point in $\Omega_{2}$, we have

$$
\begin{aligned}
\left(S-S_{1}\right) \cdot\left(S-S_{2}\right) & =\left[u-u_{1}, u-u_{2}\right]+\left\langle\phi-\phi_{1}, \gamma\left(\phi-\phi_{2}\right)\right\rangle \\
& =\left[T\left(\phi-\phi_{1}\right), u-u_{2}\right]+\left\langle\phi-\phi_{1}, \gamma\left(\phi-\phi_{2}\right)\right\rangle \\
& =\left\langle\phi-\phi_{1}, T^{*}\left(u-u_{2}\right)+\gamma\left(\phi-\phi_{2}\right)\right\rangle,
\end{aligned}
$$

by (3) and using the fact that the boundary terms vanish since $\phi_{1}=\phi=\phi_{B}$ on $B$. By (8) we therefore have

$$
\left(S-S_{1}\right) \cdot\left(S-S_{2}\right)=\left\langle\phi-\phi_{1}, f(\phi)-f\left(\phi_{2}\right)+\gamma\left(\phi-\phi_{2}\right)\right\rangle .
$$

To get a hypercircle result from this we now suppose that

$$
\frac{f\left(\phi_{i}\right)-f\left(\phi_{j}\right)}{\phi_{i}-\phi_{j}} \leqslant-\gamma \leqslant 0 \text { for all } \phi_{i}, \phi_{j},
$$

that is, $-f(\phi)$ is monotone. For differentiable $f$ this implies

$$
\frac{d f(\psi)}{d \psi} \leqslant-\gamma \leqslant 0 \text { for all } \psi .
$$

We also choose

$$
\phi_{2}=\phi_{1},
$$

which we can do. Then, under these circumstances, it follows from (10) that

$$
\left(S-S_{1}\right) \cdot\left(S-S_{2}\right) \leqslant 0 .
$$


Hence the solution $S$ lies inside (or on) a hypersphere

$$
(X-C)^{2}=R^{2},
$$

with centre $C$ and radius $R$ given by

$$
2 C=S_{1}+S_{2}, \quad 4 R^{2}=\left(S_{1}-S_{2}\right)^{2},
$$

where $S_{i}^{2}=S_{i} . S_{i}$.

We owe to the referee the observation that under the assumptions used here the geometrical formulation suggests a uniqueness proof. For if $S_{1}=S_{2}=$ one solution, then any other solution is contained in a hypersphere of radius $R=0$ about $C=S_{1}$ and hence is again $S_{1}$.

Linear problems correspond to

$$
f(\phi)=g \phi+h,
$$

where $g$ and $h$ are given functions, and we recover the hypercircle results for them by replacing $\gamma$ by $-g$ and imposing the condition $-g \geqslant 0$. In this case condition (13) becomes unnecessary and the solution $S$ actually lies on the hypersphere (15).

\section{An error bound}

From the hypersphere (15) we have

Now

$$
\left(S_{1}-S\right)^{2} \leqslant\left(S_{1}-S_{2}\right)^{2}
$$

$$
\begin{aligned}
\left(S_{1}-S\right)^{2} & =\left[u_{1}-u, u_{1}-u\right]+\left\langle\phi_{1}-\phi, \gamma\left(\phi_{1}-\phi\right)\right\rangle \\
& =\left[T\left(\phi_{1}-\phi\right), T\left(\phi_{1}-\phi\right)\right]+\left\langle\phi_{1}-\phi, \gamma\left(\phi_{1}-\phi\right)\right\rangle \\
& =\left\langle\phi_{1}-\phi,\left(T^{*} T+\gamma\right)\left(\phi_{1}-\phi\right)\right\rangle \text { by (3) } \\
& \geqslant(\Lambda+\gamma)\left\langle\phi_{1}-\phi, \phi_{1}-\phi\right\rangle,
\end{aligned}
$$

where $\Lambda$ is a lower bound to the lowest eigenvalue $\lambda_{0}$ of the problem

$$
T^{*} T \theta=\lambda \theta \text { in } V, \quad \theta=0 \text { on } B .
$$

Equations (18) and (19) give

$$
\left\langle\phi_{1}-\phi, \phi_{1}-\phi\right\rangle \leqslant \frac{\left(S_{1}-S_{2}\right)^{2}}{\Lambda+\gamma}=\frac{4 R^{2}}{\Lambda+\gamma}=E\left(\phi_{1}\right) \quad \text { say. }
$$

We have thus obtained a mean square estimate of the error in the trial function $\phi_{1}$. 


\section{Example 1}

To illustrate these results we first take the non-linear ordinary differential equation problem described by

$$
\begin{gathered}
d^{2} \phi / d x^{2}=\phi+\phi^{2} / 4, \quad-1<x<1 \\
\phi(-1)=\phi(1)=1
\end{gathered}
$$

This problem arises in heat conduction and it is known that there is a non-negative solution $\phi[4]$. Our theory applies to (22) and (23) with the choices

$$
\begin{gathered}
T=d / d x, \quad T^{*}=-d / d x, \\
f(\phi)=-\phi-\phi^{2} / 4 \\
\phi_{B}=1 \quad \text { at } \quad x= \pm 1 .
\end{gathered}
$$

If we confine our attention to non-negative functions, then

$$
-d f(\psi) / d \psi=1+\psi / 2 \geqslant 1 \text { for } \psi \geqslant 0
$$

By (12) we see therefore that we can choose

$$
\gamma=1
$$

The scalar product (9) for this problem is then

$$
S_{i} \cdot S_{j}=\int_{-1}^{1}\left(u_{i} u_{j}+\phi_{i} \phi_{j}\right) d x .
$$

We take very simple trial functions $S_{1}$ in $\Omega_{1}$ and $S_{2}$ in $\Omega_{2}$ with $S_{1}=\left(u_{1}, \phi_{1}\right)$ and $S_{2}=\left(u_{2}, \phi_{2}\right)$ where

and

$$
\begin{gathered}
\phi_{1}=1+\alpha\left(1-x^{2}\right), \\
u_{1}=d \phi_{1} / d x=-2 \alpha x,
\end{gathered}
$$

$$
\begin{gathered}
\phi_{2}=\phi_{1}, \\
u_{2}=\frac{5}{4} x+\frac{8}{2} \alpha\left(x-\frac{1}{8} x^{3}\right)+\frac{1}{4} \alpha^{2}\left(x-\frac{2}{8} x^{3}+\frac{1}{6} x^{5}\right),
\end{gathered}
$$

where $u_{2}$ is a solution of

$$
-d u_{2} / d x=f\left(\phi_{1}\right)=-\phi_{1}-\phi_{1}^{2} / 4
$$

Here $\alpha$ is a parameter which is found by minimizing the radius $R$ of the hypersphere given in this case by

$$
4 R^{2}=\left(S_{1}-S_{2}\right)^{2}=\int_{-1}^{1}\left(u_{1}-u_{2}\right)^{2} d x
$$


The results are

$$
\alpha=-0.399, \quad R=0.01944,|C|=1.23733 .
$$

Since the lowest eigenvalue of the associated eigenvalue problem (20) is $\lambda_{0}=\pi^{2} / 4$, we can choose

$$
\Lambda=\pi^{2} / 4,
$$

and so by (21) we find the mean square error

$$
E\left(\phi_{1}\right)=4 R^{2} /(\Lambda+\gamma)=0.00044
$$

in our trial function

$$
\phi_{1}=1-0.399\left(1-x^{2}\right) .
$$

Bounding curves for the exact solution $\phi$ are known, namely

and

$$
c_{1}(x) \leqslant \phi(x) \leqslant c_{2}(x),
$$

$$
b_{1}(x) \leqslant \phi(x) \leqslant b_{2}(x),
$$

where $c_{1}(x)$ and $c_{2}(x)$ are the Collatz [5] curves

$$
\begin{aligned}
& c_{1}(x)=1-0.35\left(1-x^{2}\right)-0.05\left(1-x^{4}\right), \\
& c_{2}(x)=1-0.35\left(1-x^{2}\right)-0.04\left(1-x^{4}\right),
\end{aligned}
$$

and $b_{1}(x)$ and $b_{2}(x)$ are the Bailey et al. [4] curves

$$
\begin{aligned}
& b_{1}(x)=\operatorname{sech} \sqrt{\frac{3}{2}} \cosh \sqrt{\frac{8}{2}} x, \\
& b_{2}(x)=\operatorname{sech} 1 \cosh x .
\end{aligned}
$$

Our trial solution $\phi_{1}$ is compared with these curves in Table 1 . We see that $\phi_{1}$ lies between $b_{1}(x)$ and $b_{2}(x)$, but strays just above $c_{2}(x)$ at $x= \pm 0.6$ and $x= \pm 0.8$. A more accurate approximate solution could be obtained here by taking, for

TABLE 1

Hypercircle function $\phi_{1}=1-0.399\left(1-x^{2}\right)$ and bounding curves for the exact solution, Example 1

\begin{tabular}{rlllll}
\hline \multicolumn{1}{c}{$x$} & $b_{1}(x)$ & $c_{1}(x)$ & $\phi_{1}(x)$ & $c_{2}(x)$ & $b_{2}(x)$ \\
\hline 0.0 & 0.5409 & 0.6000 & 0.6010 & 0.6100 & 0.6480 \\
\pm 0.2 & 0.5572 & 0.6140 & 0.6170 & 0.6241 & 0.6611 \\
\pm 0.4 & 0.6071 & 0.6572 & 0.6648 & 0.6670 & 0.7006 \\
\pm 0.6 & 0.6937 & 0.7324 & 0.7446 & 0.7412 & 0.7682 \\
\pm 0.8 & 0.8220 & 0.8444 & 0.8564 & 0.8504 & 0.8667 \\
\pm 1.0 & 1.0 & 1.0 & 1.0 & 1.0 & 1.0 \\
\hline
\end{tabular}


example,

$$
\phi_{1}=1+\alpha\left(1-x^{2}\right)+\beta\left(1-x^{4}\right),
$$

but the simple choice in (28) is sufficient to illustrate the method.

\section{Example 2}

As our second illustrative example we consider the non-linear boundary value problem described by

$$
\begin{aligned}
\nabla^{2} \phi & =k e^{\phi} \quad \text { in } V, \\
\phi & =1 \quad \text { on } B,
\end{aligned}
$$

where $V$ is the disc $r^{2}=x^{2}+y^{2} \leqslant 1$, so that $B$ is given by $r=1$. Here $k$ is a positive constant. In this case we take

$$
\begin{gathered}
T=\operatorname{grad}, \quad T^{*}=-\operatorname{div}, \quad f(\phi)=-k e^{\phi}, \\
\phi_{B}=1 .
\end{gathered}
$$

Since

$$
-d f(\psi) / d \psi=k e^{\psi} \geqslant 0 \text { for all } \psi \text { and } k \geqslant 0
$$

we see by (12) that we can choose

$$
\gamma=0
$$

The scalar product (9) for this problem is then

$$
S_{i} \cdot S_{j}=\int_{V} u_{i} u_{j} d V,
$$

and thus we are working in the $F$-space $H_{u}$ rather than the space $H_{u} \times H_{\phi}$.

We shall seek the radially symmetric solution of (37) and (38) approximated by

$$
\phi_{1}=1+\beta\left(r^{2}-1\right)
$$

where $\beta$ is an arbitrary parameter. Then

and

$$
\mathbf{u}_{1}=\operatorname{grad} \phi_{1}=2 \beta r \hat{r}
$$

which has solution

$$
-\operatorname{div} \mathbf{u}_{2}=f\left(\phi_{1}\right)=-k \phi^{\phi_{1}} \text {, }
$$

$$
\mathbf{u}_{2}=\frac{k e^{1-\beta}}{2 \beta r}\left(e^{\beta r^{2}}-1\right) \hat{f}
$$


finite at the origin. From (16) and (42) we find that

$$
4 R^{2}=\iint\left(u_{1}-u_{2}\right)^{2} r d r d \theta=2 \pi \int_{0}^{1}\left(u_{1}-u_{2}\right)^{2} r d r,
$$

and by integration

$$
2 R^{2} / \pi=\beta^{2}+\hat{k}\left\{e^{i-\hat{\rho}}\left(\overline{1}+\hat{\beta}^{-1}\right)-e \hat{\beta}^{-1}\right\}+\left(k^{2} \hat{\beta}^{-2} e^{2-2 \hat{\beta} / 8)} \int_{0}^{\beta} t^{-1}\left(e^{\hat{L}-1}\right)^{2} d t .\right.
$$

Since the lowest eigenvalue of the associated eigenvalue problem (20) is $\lambda_{0}=\pi^{2} / 4$, we can choose

$$
\Lambda=\pi^{2} / 4,
$$

and so by (21) we have the mean square error estimate

$$
E\left(\phi_{1}\right)=16 R^{2} / \pi^{2} \text {. }
$$

The optimum value of $\beta$ is found by minimizing the radius $R$ of the hypersphere as given by equation (48), and the results for three values of the constant $k$ are given in Table 2. Evidently better approximations to the solution are given by $\phi_{1}$

TABLE 2

Hypersphere parameters, Example 2

\begin{tabular}{ccccc}
\hline$k$ & \multicolumn{1}{c}{$\beta$} & \multicolumn{1}{c}{$R$} & $|C|$ & \multicolumn{1}{c}{$E\left(\phi_{1}\right)$} \\
\hline 5 & 1.405 & 0.31831 & 3.5437 & 0.16415 \\
2 & 0.8065 & 0.10137 & 2.02708 & 0.01666 \\
1 & 0.4926 & 0.03698 & 1.23547 & 0.002217 \\
\hline
\end{tabular}

as $k$ becomes smaller, since the radius $R$ of the hypersphere then decreases, as does the parameter $\beta$. This situation is not surprising since $\phi_{1}=1$ corresponds to the exact solution, corresponding to zero hypersphere radius, of the problem $\nabla^{2} \phi=0, \phi=1$ on $B$, for the unit disc.

\section{Concluding remarks}

We have presented a geometrical approach to the problem of solving a certain class of non-linear boundary value problems, thereby extending the available hypercircle results for linear problems. Alternative variational results exist for these problems-in the linear case they are directly related to the geometrical results [3], while for the non-linear case it will be seen from [1] that there is no such direct connection. 
Also, we remark that while the error estimates in our examples are of the meansquare type, point wise error bounds can be established for examples 1 (see [2]) and 2.

\section{Acknowledgements}

We are greatly indebted to Professor J. L. Synge, F.R.S., for a most stimulating correspondence on this work. The second author also thanks Mr. D. D. McGregor and Dr. H. B. Thompson for useful discussions.

\section{References}

[1] A. M. Arthurs, Complementary variational principles (Oxford: Clarendon Press, 1970), Chapter 4.

[2] A. M. Arthurs, "Dual extremum principles and error bounds for a class of boundary value problems", J. Math. Anal. Appl. 41 (1973), 781-795.

[3] A. M. Arthurs, "On variational principles and the hypercircle for boundary value problems", Proc. Roy. Irish Acad. 77A (1977), 75-83.

[4] P. B. Bailey, L. F. Shampine and P. E. Waltman, Nonlinear two-point boundary value problems (New York: Academic Press, 1968), p. 117

[5] L. Collatz, "Aufgaben monotoner Art", Arch. Math. 3 (1952), 366-376.

[6] B. Noble and M. J. Sewell, "On dual extremum principles in applied mathematics", J. Inst. Maths. and Applics. 9 (1972), 124-193.

[7] J. L. Synge, The hypercircle in mathematical physics (Cambridge University Press, 1957).

Department of Mathematics

University of York

York, YO1 5DD

England

and

Department of Mathematics

University of Queensland

St. Lucia, Q 4067

Australia 